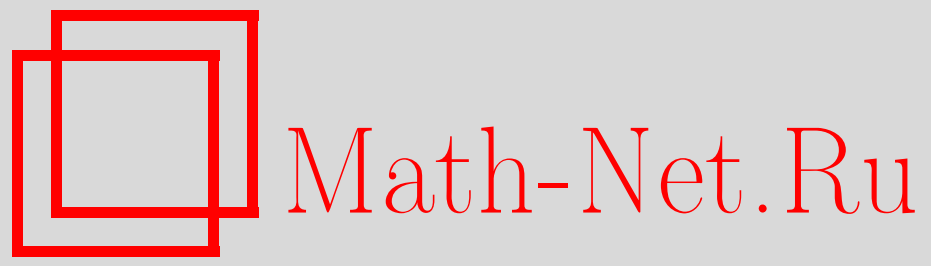

А. А. Панов, О разнообразии отображений Пуанкаре для кубических уравнений с переменными коэффициентами, Функи. анализ и его прил., 1999, том 33, выпуск 4, 84-88

DOI: https://doi.org/10.4213/faa390

Использование Общероссийского математического портала MathNet.Ru подразумевает, что вы прочитали и согласны с пользовательским соглашением http://www. mathnet.ru/rus/agreement

Параметры загрузки:

IP: 52.87 .193 .239

26 апреля 2023 г., 14:50:45

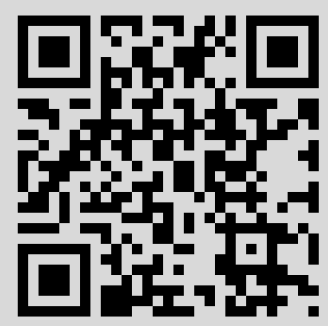


Каждый элемент нижней части матрицы $A J$ сопряжен центрально симметричному элементу верхней части. Значит,

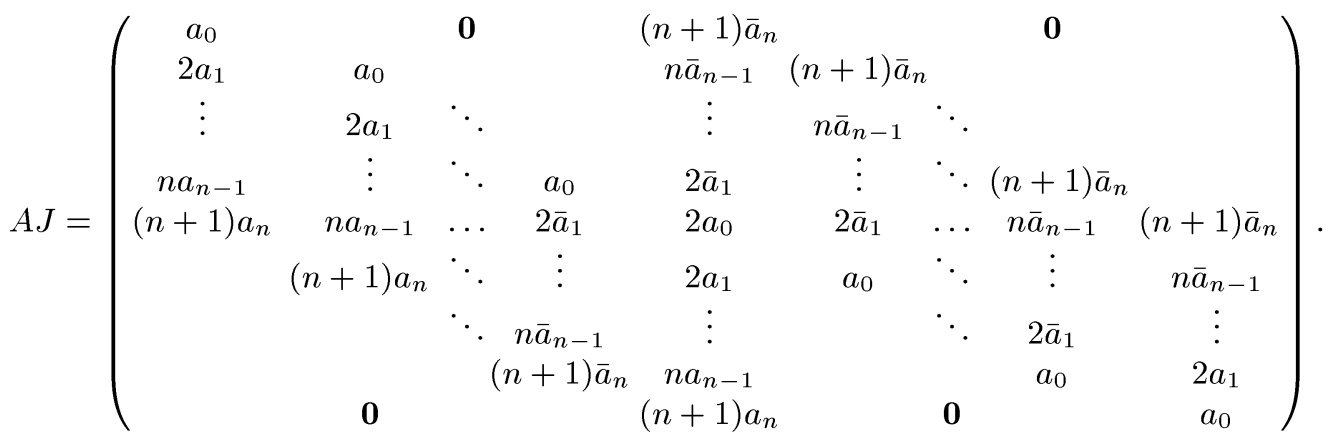

Умножая $A J$ справа на $M$, мы заменяем средний столбец на $\left(0, \ldots, 0, a_{0}, 2 a_{1}, \ldots\right.$, $\left.(n+1) a_{n}\right)^{t}$ и получаем матрицу с верхней строкой $\left(a_{0}, 0, \ldots, 0\right)$, и дополнительный минор к $a_{0}$ - транспонированная матрица Сильвестра для результанта многочленов $a_{0}+2 a_{1} t+\cdots+(n+1) a_{n} t^{n}$ и $(n+1) \bar{a}_{n}+n \bar{a}_{n-1} t+\cdots+\bar{a}_{0} t^{n}$. Значит, $\operatorname{det} A \operatorname{det} J \operatorname{det} M=a_{0} \operatorname{Res}_{t}\left(q^{\prime}, t^{n} \bar{q}^{\prime}\left(t^{-1}\right)\right)$.

Ясно, что $\operatorname{det} M=\frac{1}{2}$ и $A_{k, k}=a_{0}^{-k}$, а следовательно, $\operatorname{det} A=\prod A_{k, k}^{2}=a_{0}^{-n(n+1)}$. Осталось заметить, что обращение порядка элементов $\partial a_{m}$ в $J$ меняет знак таким же образом, как и перестановка аргументов результанта.

Я благодарю П. Этингофа и Н. Г. Кружилина за полезные обсуждения.

\title{
ЛиТЕРАТУРА
}

1. Прилепко А. И. Потенциала теории обратные задачи. Математическая энциклопедия. Т. 4, Советская энциклопедия, 1984, с. 520-523.

\section{О разнообразии отображений Пуанкаре для кубических уравнений с переменными коэффициентами*}

\author{
(C) 1999. А. А. ПАнов
}

1. Введение. Основным объектом нашего изучения является уравнение вида

$$
\dot{x}=a_{3}(t) x^{3}+a_{2}(t) x^{2}+a_{1}(t) x+a_{0}(t), \quad t \in[0,1], a_{i} \in C^{1} .
$$

Введем несколько стандартных обозначений. Рассмотрим уравнение вида

$$
\dot{x}=h(x, t), \quad h \in C^{1} .
$$

*Работа частично поддержана грантами РФФИ 95-01-01258, CRDF RM1-229, INTAS 93-05-07. 
Пусть $\varphi(x, t)$ - решение этого уравнения с начальным условием $\varphi(x, 0)=x$. Обозначим через $P^{T}[h]$ отображение последования этого уравнения за время $T$, $P^{T}[h]: x \rightarrow \varphi(x, T)$; иногда вместо $h$ ставится его явное выражение.

Можно поставить вопрос, сколько невырожденных периодических решений может иметь уравнение (1). Циклическим решением уравнения (1) будем называть решения, принимающие одинаковые значения в 0 и 1. Известно [1], что при $a_{3}(t)=a_{2}(t) \equiv 0$ уравнение $(1)$ может иметь не более двух, при $a_{3}(t) \equiv 0-$ не более трех, а при $a_{3}(t)>0$ - не более четырех невырожденных циклических решений. Однако Л. Нето [2] показал, что при общих $a_{3}(t)$ уравнение $(1)$ может иметь сколь угодно большое число невырожденных циклических решений.

Конструкция Нето, позволяющая строить уравнения вида (1) со сколь угодно большим числом невырожденных циклических решений, состоит в следующем. Рассматривается уравнение вида $\dot{x}=a_{2}(t) x^{2}$, для которого отображение $P^{1}\left[a_{2}(t) y^{2}\right]$ тождественно на некотором малом отрезке $G$, содержащем 0 . Далее уравнение возмушается с помощью добавки $\varepsilon a_{3}(t) x^{3}$, где $\varepsilon$ мало, и показывается, что за счет выбора $a_{3}(t)$ можно получить сколь угодно много невырожденных циклических решений на отрезке $G$. Таким образом, видно, что в конструкции Нето отображение $P^{1}\left[\varepsilon a_{3}(t) y^{3}+a_{2}(t) y^{2}\right]$ близко к тождественному. Нас будут интересовать свойства всевозможных отображений последования $P^{1}\left[a_{3}(t) y^{3}+\cdots+a_{0}(t)\right]$.

Оказывается, что множество функций последования уравнения (1) всюду плотно в множестве непрерывных монотонных функций.

ТЕОРЕмА 1. Для любого отрезка множество отображений последования уравнения (1) за время 1 всюду плотно в пространстве сохраняющих ориентацию гомеоморфизмов этого отрезка на свой образ.

СлЕДСТВИЕ. Уравнение вида (1) может иметь любое число ииклических решений.

Выражаю признательность Ю. С. Ильяшенко за постановку задач, регулярные консультации и всемерную поддержку. Также выражаю благодарность Д. А. Панову за многочисленные обсуждения этой работы.

\section{2. Леммы о композициях одномерных отображений.}

Лемма 1. Рассмотрим уравнение вида

$$
\dot{x}=a_{n} x^{n}+a_{n-1} x^{n-1}+\cdots+a_{0}, \quad a_{i} \in \mathbb{R} .
$$

Тогда для любого отрезка множество отображений последования уравнения (3) за время 1 всюду плотно в пространстве сохраняюших ориентацию гомеоморфизмов отрезка на свой образ.

ТЕорема 2. Пусть отображение последования $P^{1}\left[a_{n} y^{n}+\cdots+a_{0}\right], a_{i} \in \mathbb{R}$, уравнения (3) определено на отрезке $[a, b]$. Тогда для любого $\varepsilon>0$ существует уравнение вида (1), такое, что на отрезке $[a, b]$ отображения последования уравнений (1) и (2) за время 1 отличаются не более чем на в.

Теорема 1 следует из леммы 1 и теоремы 2.

Лемма 2. Рассмотрим уравнение

$$
\dot{x}=h(x) .
$$

Пусть задань отрезки $[a, b] u\left[a^{\prime}, b^{\prime}\right]$, такие, что $[a, b] \subset\left(a^{\prime}, b^{\prime}\right)$, и отображение последования $P^{T}[h]$ этого уравнения определено на отрезке $\left[a^{\prime}, b^{\prime}\right]$. Пусть 
$\max _{t \in[0, T]} \varphi\left(b^{\prime}, t\right)=B u \min _{t \in[0, T]} \varphi\left(a^{\prime}, t\right)=A$. Пусть, кроме того, существует семейство функций $H_{\varepsilon}$, определенных на отрезке $[A, B]$, такое, ито на этом отрезке $\left|H_{\varepsilon}-P^{\varepsilon}[h]\right|<C \varepsilon^{3 / 2}$. Пусть $G_{n}=H_{1 / n} \circ \cdots \circ H_{1 / n}$. Тогда $G_{n}$ равномерно стремится $к P^{T}[h]$ на отрезке $[a, b]$.

Лемма 3. Пусть $\dot{x}=g(x)$ и $\dot{x}=f(x)$ - векторные поля на $\mathbb{R}^{1}$ u $h=[f, g]$ их коммутатор. Пусть отображения последования $P^{1}[g]$ и $P^{1}[f]$ определень на некотором отрезке $[a, b]$. Тогда при $\varepsilon \rightarrow 0$

$$
P^{\varepsilon}[f](x) \circ P^{\varepsilon}[g](x) \circ P^{-\varepsilon}[f](x) \circ P^{-\varepsilon}[g](x)=x+\varepsilon^{2} h(x)+F(\varepsilon, x),
$$

әде $F(\varepsilon, x)$ есть $O\left(\varepsilon^{3}\right)$.

Доказательства лемм 1, 2 и 3 просты и здесь не приводятся.

\section{3. Основная лемма.}

Лемма 4. Для любого $n$ и любого отрезка $[A, B]$ существуют $\varepsilon_{0}>0, C>0$ и уравнение вида (1), такие, ито для любого $\varepsilon \in\left[0, \varepsilon_{0}\right]$ на отрезке $[A, B]$ отображения последования уравнения (1) и уравнения

$$
\dot{x}=x^{n}
$$

за время $\varepsilon$ отличаются не более чем на $C \varepsilon^{3 / 2}$.

ЗАмечАниЕ. Основная идея леммы состоит в использовании следующего факта. Коммутатор $\left[x^{n}, x^{2}\right]$ равен $-(n-2) x^{n+1}$; таким образом, если $n>2$, то, последовательно беря коммутаторы получившихся полей с квадратичным полем, мы можем получить в итоге любую степень $x$.

ДокАЗАТЕльство леммы 4 проведем по индукции относительно степени $n$.

База индукиии. Мы будем строить полином третей степени с зависящими от времени коэффициентами так, чтобы на некотором отрезке $[A, B]$ отображение последования $P^{\varepsilon}\left[a_{3}(t) y^{3}+\cdots+a_{0}(t)\right]$ уравнения (1) приближало отображение последования $P^{\varepsilon}\left[y^{4}\right]$ уравнения $(4)$ с точностью $\varepsilon^{3 / 2}$.

Для этого сначала определим $\varepsilon_{0}>0$ так, чтобы для любого $\varepsilon<\varepsilon_{0}$ отображения последования уравнений (4) при $n=2,3,4$ за время $16 \sqrt{\varepsilon}$ были определены на отрезке $[A, B]$.

Теперь определим разрывное векторное поле $h(x, t)$. Для этого рассмотрим область $[A, B] \times[0, \varepsilon]$ и разобьем ее на четыре части $R_{1}, R_{2}, R_{3}, R_{4}$,

$$
R_{i}=[A, B] \times\left[\frac{1}{4}(i-1) \varepsilon, \frac{1}{4} i \varepsilon\right], \quad i=1,2,3,4 .
$$

Пусть $h \mid R_{i}=h_{i}$, где

$$
h_{1}(x, t)=4 x^{3} / \sqrt{\varepsilon}, \quad h_{2}(x, t)=4 x^{2} / \sqrt{\varepsilon}, \quad h_{3}(x, t)=-4 x^{3} / \sqrt{\varepsilon}, \quad h_{4}(x, t)=-4 x^{2} / \sqrt{\varepsilon} .
$$

Кроме того, определим разрывное векторное поле $h^{*}(x, t)$. Для этого рассмотрим область $[A, B] \times[0, \sqrt{\varepsilon}]$ и разобьем ее на четыре части $G_{1}, G_{2}, G_{3}, G_{4}$, где

Пусть $H^{*} \mid G_{i}=h_{i}^{*}$, где

$$
G_{i}=[A, B] \times\left[\frac{1}{4}(i-1) \sqrt{\varepsilon}, \frac{1}{4} i \sqrt{\varepsilon}\right], \quad i=1,2,3,4 .
$$

$$
h_{1}(x, t)=4 x^{3}, \quad h_{2}(x, t)=4 x^{2}, \quad h_{3}(x, t)=-4 x^{3}, \quad h_{4}(x, t)=-4 x^{2} .
$$

Воспользовавшись тождеством $P^{\varepsilon}[h(y)]=P^{\sqrt{\varepsilon}}[\sqrt{\varepsilon} h(y)]$, мы получим, что отображение последования поля $h(x, t)$ за время $\varepsilon$ совпадает с отображением последования за время $\sqrt{\varepsilon}$ поля $h^{*}(x, t)$. 
Применяя лемму 3 , получим, что в этом случае на отрезке $[A, B]$ отображение последования поля $h^{*}(x, t)$ за время $\sqrt{\varepsilon}$ задается формулой

$$
P^{\sqrt{\varepsilon}}\left[h^{*}\right](x)=x+(\sqrt{\varepsilon} / 4)^{2}\left[4 x^{3}, 4 x^{2}\right]+F_{1}(\varepsilon, x),
$$

где $F_{1}(\varepsilon, x)<C_{1} \varepsilon^{3 / 2}$ на $[A, B]$ для малых $\varepsilon$.

Таким образом, отображение последования для поля $h(x, t)$ за время $\varepsilon$ имеет вид

$$
P^{\varepsilon}[h](x)=x+\varepsilon x^{4}+F_{1}(\varepsilon, x) .
$$

Заметим также, что отображение последования уравнения $\dot{x}=x^{4}$ за время $\varepsilon$ выражается формулой

$$
P^{\varepsilon}\left[y^{4}\right](x)=x+\varepsilon x^{4}+F_{2}(\varepsilon, x), \quad \text { где } F_{2}(\varepsilon, x)<C_{2} \varepsilon^{2} \text { на }[A, B] \text { для малых } \varepsilon .
$$

Теперь окончательно получаем, что на отрезке $[A, B]$

$$
\left|P^{\varepsilon}[h](x)-P^{\varepsilon}\left[y^{4}\right](x)\right|<C_{3} \varepsilon^{3 / 2}
$$

для достаточно малых $\varepsilon$, причем $C_{3}$ зависит только от $[A, B]$.

Для того чтобы завершить доказательство леммы для случая $n=4$, заметим, что, несмотря на разрывность кубического поля $h(x, t)$, мы можем сгладить коэффициенты $a_{i}(t)$ так, чтобы поле стало гладким и неравенство (5) осталось в силе.

Шаг индукиии. Итак, пусть мы умеем строить уравнение вида (1), отображение последования которого на заранее выбранном отрезке приближает отображение последования уравнения $\dot{x}=x^{n-1}$ за время $\varepsilon$ с точностью $\varepsilon^{3 / 2}$. Пусть отображение последования за время $T$ определено на отрезке $[a, b]$. Тогда по лемме 2 мы умеем строить уравнение вида (1), отображение последования которого приближает отображение последования уравнения $\dot{x}=x^{n-1}$ за время $T$ с любой наперед заданной точностью.

Теперь, если мы повторим схему, использованную в доказательстве базы индукции, заменяя поле $\dot{x}=x^{4}$ на $\dot{x}=x^{n-1}$, то окончательно докажем лемму.

\section{4. Доказательство теоремы о плотности.}

ДоКАЗАТЕЛЬСтво тЕОРЕмы 2. Итак, мы имеем полиномиальное дифференциальное уравнение

$$
\dot{x}=a_{n} x^{n}+a_{n-1} x^{n-1}+\cdots+a_{0},
$$

причем отображение последования $P^{1}\left[a_{n} y^{n}+a_{n-1} y^{n-1}+\cdots+a_{0}\right]$ этого уравнения определено на отрезке $[a, b]$. Выберем отрезок $\left[a^{\prime}, b^{\prime}\right]$, на котором также определено отображение последования и для которого выполняется условие $[a, b] \subset\left(a^{\prime}, b^{\prime}\right)$. Теперь найдем отрезок $[A, B]$ из условия леммы 2 . В силу леммы 3 для любого достаточно малого $\varepsilon$ существуют такие кубические поля $h_{n}(x)$, что отображение последования уравнения $\dot{x}=h_{n}(x)$ за время $\varepsilon /(n+1)$ на отрезке $[A-1, B+1]$ приближает отображение последования уравнения $\dot{x}=(n+1) a_{n} x^{n}$ с точностью $\varepsilon^{3 / 2}$, т.е.

$$
\left|P^{\varepsilon /(n+1)}\left[h_{n}\right](x)-P^{\varepsilon /(n+1)}\left[(n+1) a_{n} y^{n}\right](x)\right|<C_{4} \varepsilon^{3 / 2},
$$

где $C_{4}$ зависит только от $[A, B]$ и $n$.

Теперь рассмотрим разрывное векторное поле $H_{\varepsilon}^{*}(x)$, равное в области $[A-1, B+1] \times[i \varepsilon /(n+1),(i+1) \varepsilon /(n+1)]$ полю $h_{i}(x)$, где $i=1, \ldots, n+1$. Тогда 
получим, что на отрезке $[A, B]$ отображение последования уравнения $\dot{x}=H_{\varepsilon}^{*}(x)$ за время $\varepsilon$ равняется

$$
P^{\varepsilon}\left[H_{\varepsilon}^{*}\right](x)=x+\varepsilon a_{n} x^{n}+\cdots+\varepsilon a_{0}+F_{5}(x, \varepsilon),
$$

где $F_{5}(x, \varepsilon)<C_{5} \varepsilon^{3 / 2}$, причем $C_{5}$ зависит только от $[A, B]$ и $n$. Таким образом, на отрезке $[A, B]$ имеем неравенство

$$
\left|P^{\varepsilon}\left[H_{\varepsilon}^{*}\right](x)-P^{\varepsilon}\left[a_{n} y^{n}+a_{n-1} y^{n-1}+\cdots+a_{0}\right]\right|<C_{5} \varepsilon^{3 / 2} .
$$

Итак, нам осталось только сгладить наше векторное поле $H_{\varepsilon}^{*}(x)$, как мы это уже делали раньше, при условии, что сохраняется неравенство (6). Применяя к получившемуся полю $H_{\varepsilon}(x)$ лемму 2 , окончательно докажем теорему 2.

\title{
ЛИТЕРАТУРА
}

1. Плисс В. А. ДАН СССР, 127, № 5, 965-968 (1959). 2. Neto A. L. Invent. Math., 59, No. 2, 67-76 (1980).

Московский государственный университет им. М. В. Ломоносова

Поступило в редакцию 23 мая 1997 г. В переработанном виде 7 мая 1998 г.

УДК 513.6

\section{Бирациональные автоморфизмы двойных накрытий Фано*}

\author{
(c) 1999. А. В. Пухликов
}

1. В настоящей заметке установлена бирациональная сверхжесткость двойных гиперповерхностей Фано индекса один. Напомним [2], что гладкое многообразие Фано $V$ называется бирационально сверхжестким, если для любого бирационального отображения $\chi: V \rightarrow V^{\prime}$ на унилинейчатое многообразие той же размерности и линейной системы дивизоров $\Sigma^{\prime}$ на $V^{\prime}$ без неподвижных компонент выполнено условие монотонности $c(D, V) \leqslant c\left(D^{\prime}, V^{\prime}\right)$, где $D^{\prime} \in \Sigma^{\prime}$, $D \in \Sigma=\left(\chi^{-1}\right)_{*} \Sigma^{\prime}$, т. е. $\Sigma$ (собственный прообраз системы $\Sigma^{\prime}$ относительно $\left.\chi\right)$ - линейная система дивизоров на $V$ без неподвижных компонент, а $c(Y, X)$ для неособого в коразмерности 1 многообразия $X$ и дивизора Вейля $Y$ на нем обозначает порог канонического присоединения,

$$
c(Y, X)=\sup \left\{b / a\left|b \in \mathbb{Z}_{+}, a \in \mathbb{Z}_{+} \backslash\{0\},\right| a Y+b K_{X} \mid \neq \varnothing\right\} .
$$

Зафиксируем целые числа $M \geqslant 4, m \geqslant 3$ и $l \geqslant 1$, удовлетворяющие равенству $m+l=M+1$. Символом $\mathbb{P}$ обозначим проективное пространство $\mathbb{P}^{M+1}$ над полем комплексных чисел $\mathbb{C}$. Пусть $Q=Q_{m} \subset \mathbb{P}$ и $W^{*}=W_{2 l}^{*} \subset \mathbb{P}$ - гиперповерхности степеней $m$ и $2 l$ соответственно, а $\sigma: V \rightarrow Q-$ двойное накрытие, разветвленное над $W=W^{*} \cap Q$. Многообразие $V$ есть многообразие Фано размерности $M$ с группой Пикара $\mathbb{Z} K_{V}$. Наш основной результат - следующая

*Работа выполнена при поддержке гранта 99-15-96013 поддержки исследований молодых российских ученых-докторов наук. 